\title{
A generalized solution for groundwater head fluctuation in a tidal leaky aquifer system
}

\author{
Mo-Hsiung Chuang ${ }^{1}$ and Hund-Der Yeh ${ }^{2, *}$ \\ ${ }^{1}$ Department of Urban Planning and Disaster Management, Ming-Chuan University, \\ Gweishan District, Taoyuan, Taiwan. \\ ${ }^{2}$ Institute of Environmental Engineering, National Chiao Tung University, Hsinchu, Taiwan. \\ ${ }^{*}$ Corresponding author.e-mail: hdyeh@mail.nctu.edu.tw
}

\begin{abstract}
A new analytical solution is developed for describing groundwater level fluctuations in a coupled leaky confined aquifer system which consists of an unconfined aquifer, confined aquifer, and an aquitard in between. The aquifer system has a tidal boundary at the seashore, a no flow boundary at remote inland side, and a confined aquifer extending under the sea and terminated with an outlet-capping. This new solution has shown to be a generalisation of most existing analytical solutions for a tidal aquifer system which includes single confined and leaky confined aquifers. In addition, the solution is used to explore the influences of the dimensionless leakance of the outlet-capping, the dimensionless hydraulic diffusivities, and the leakages of the inland and offshore aquitards on the head responses in the leaky confined aquifer.
\end{abstract}

\section{Introduction}

In most of the coastal areas, groundwater and seawater are hydraulically connected. The tidal dynamics in coastal aquifers is an interesting topic for hydrologists and plays an important role in numerous environmental issues in coastal areas. Jacob (1950) introduced a simple coastal aquifer system that considered a vertical beach, straight coastline and one-dimensional flow in a coastal confined aquifer. Since then, the dynamic interaction between groundwater and seawater has attracted much attention. The coastal subsurface formation is usually complex; the Jacob's solution was in fact too simple to be applied to many real-world problems. Therefore, the analytical solutions for complicated tidal aquifer configurations were developed. Those include a three-layered coastal aquifer system (Jiao and Tang 1999; Li and Jiao 2001a; Jeng et al 2002; Li et al 2007; Chuang et al 2010), a confined aquifer extending under the sea (Van der
Kamp 1972; Li and Jiao 2001b; Chuang and Yeh 2007, 2008; Li et al 2008) and a coastal confined aquifer with an outlet-capping (Guo et al 2007; Xia et al 2007; Geng et al 2009). Previous studies showed that dynamic effect of the unconfined aquifer on the head fluctuations in the confined aquifer plays an active role in solving coastal leaky confined aquifer problems.

This paper develops a new analytical solution for describing groundwater level fluctuation in a heterogeneous leaky confined aquifer extending finite distance under the sea and terminated at an outletcapping. Most of the existing analytical solutions of the tidal aquifer systems are shown as special cases of the present solution. These solutions include one-dimensional flow in a coastal confined aquifer (Jacob 1950; Ferris 1951; Van der Kamp 1972; Geng et al 2009) and a variety of coastal leaky confined aquifer systems (e.g., Jiao and Tang 1999; Li and Jiao 2001b; Chuang and Yeh 2007, 2008; Li et al 2007; Xia et al 2007; Jeng et al 2002).

Keywords. Aquitard; coastal aquifer; leaky aquifer; analytical solution. 
In addition, the present solution can be considered as an extension of Xia et al's (2007) solution with differences in two following aspects:

(1) the offshore and inland parts of the aquifer have different hydraulic properties, and

(2) the water table in the unconfined aquifer fluctuates with tide.

The influence of those two situations on the behaviour of the groundwater level fluctuation in the inland part of the confined aquifer is investigated. The joint dynamic effects of water table fluctuation, the leakage through its submarine outlet-capping, and the leakages of the inland and offshore aquitards on the head fluctuations in the inland part of the leaky confined aquifer are also examined.

\section{Problem formulation}

Consider a coastal aquifer system with an unconfined aquifer, a confined aquifer, and an aquitard between them as displayed in figure 1 . The origin of the $x$-axis is located at the intersection of the mean sea surface and the beach face. The $x$-axis is horizontal, positive landward and perpendicular to the coastal line. Tidal fluctuations in both unconfined and confined aquifers are considered. These two aquifers interact with each other through leakage. The unconfined aquifer terminates at the coast while the aquitard and confined aquifer extend over a finite distance $(l)$ under the sea with an outletcapping (Xia et al 2007). The bottom of the confined aquifer is impermeable and the leakages of the offshore and inland aquitards are different. Consider that the hydraulic parameters of the unconfined aquifer as well as the offshore and inland confined aquifers are all different. In addition, the thickness of the unconfined aquifer is very large when compared to the amplitude of the tidal fluctuation. The flow in the confined aquifer is essentially horizontal and there is a vertical leakage through the aquitard. The initial hydraulic head in the whole system is uniform and equals $h_{\mathrm{MSL}}$, which is the distance from the groundwater level to any arbitrary location. Assume that elastic storage of the aquitard is negligible and the leakage is linearly proportional to the difference in head between the unconfined aquifer and its underlain confined one.

\subsection{Groundwater flow equations}

Under above assumptions, the governing equations of the head fluctuations for the inland unconfined and confined aquifers $(x>0)$ are respectively (Chuang and Yeh 2008)

$$
\begin{aligned}
& S_{1} \frac{\partial h_{1}}{\partial t}=T_{1} \frac{\partial^{2} h_{1}}{\partial x^{2}}+L_{i}\left(h_{2}-h_{1}\right) \\
& S_{2} \frac{\partial h_{2}}{\partial t}=T_{2} \frac{\partial^{2} h_{2}}{\partial x^{2}}+L_{i}\left(h_{1}-h_{2}\right)
\end{aligned}
$$

and for the offshore aquifer $(x<0)$ is

$$
S_{3} \frac{\partial h_{2}}{\partial t}=T_{3} \frac{\partial^{2} h_{2}}{\partial x^{2}}+S_{3} T_{e} \frac{d h_{s}}{d t}+L_{o}\left(h_{s}-h_{2}\right)
$$

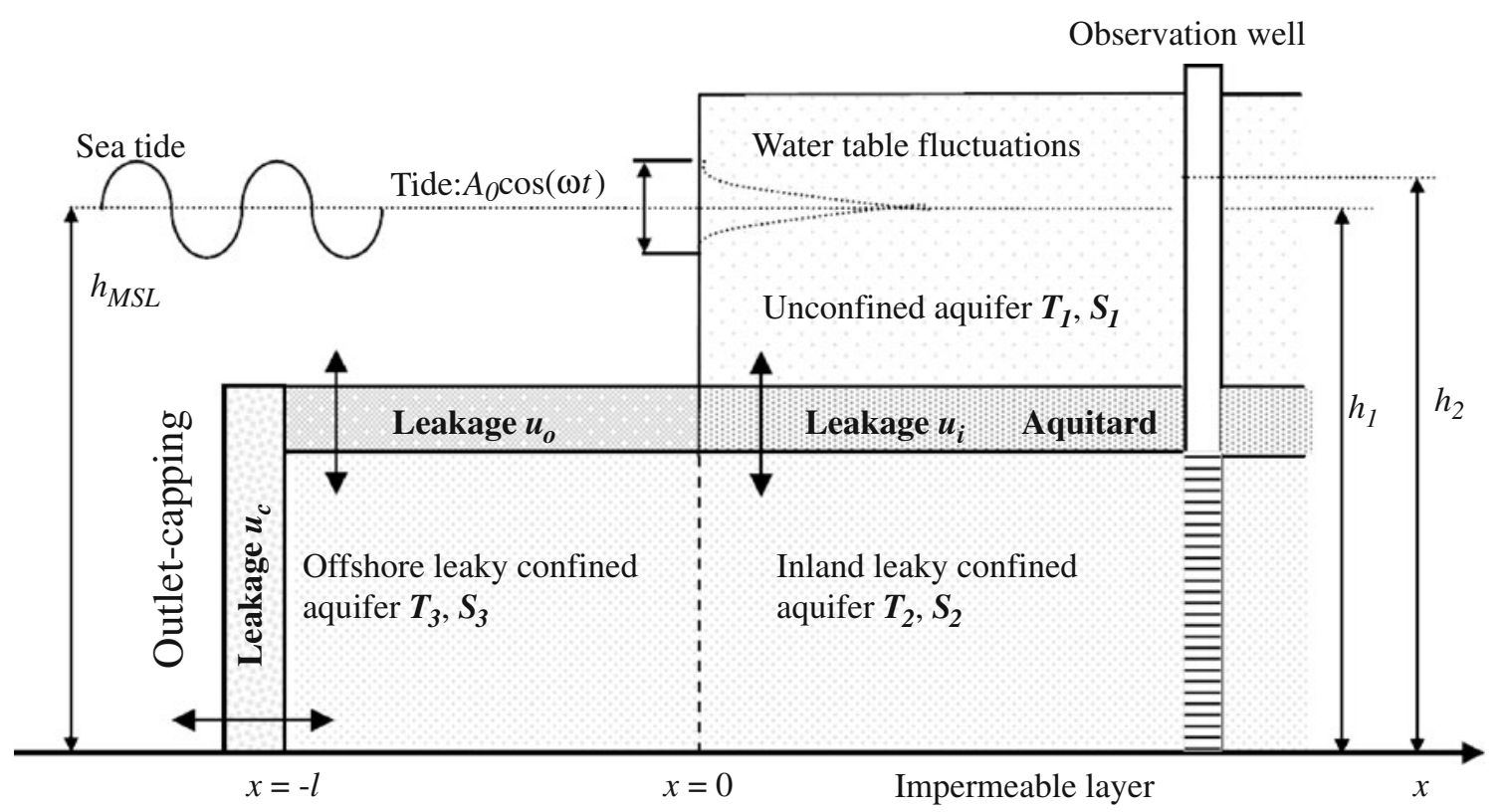

Figure 1. Schematic diagram of a tidal leaky aquifer system. 
where $h_{1}$ and $h_{2}$ are the hydraulic heads in the unconfined and confined aquifers, respectively; $h_{s}$ is the water level of the sea tide; $T_{e}$, the tidal efficiency, reflects the fluctuation of groundwater level caused by compression of both the aquifer skeleton and groundwater due to the tidal loading above the offshore aquitard (Li and Jiao 2001b); $S_{1}$ is the specific yield of the unconfined aquifer. $S_{2}$ and $S_{3}$ are the storativities of the inland and offshore confined aquifers, respectively. $T_{1}, T_{2}$ and $T_{3}$ are the transmissivities of the unconfined, inland and offshore confined aquifers, respectively. The leakage is defined as the ratio of the hydraulic conductivity of the aquitard to the thickness of the aquitard and $L_{o}$ and $L_{i}$ are the leakages of the offshore and inland aquitards, respectively. The hydraulic conductivity and/or thickness of the inland aquitard may differ from those of the offshore aquitard due to different depositional sediment faces.

\subsection{Boundary and continuity conditions}

The tidal boundary at $x=0$ may be written as:

$$
h_{1}(0, t)=h_{s}(t)=h_{\mathrm{MSL}}+A_{0} \cos (\omega \cdot t)
$$

where $h_{1}(0, t)$ is the hydraulic head at $x=0, A_{0}$ is the amplitude of the tidal change, and $\omega$ is the tidal frequency. Also $\omega=2 \pi / t_{0}$ where $t_{0}$ is the tidal period. The leakage rate at the outlet-capping is expressed as:

$-K_{3} \frac{\partial h_{2}(x, t)}{\partial x}=\frac{K^{\prime}\left(h_{s}-h_{2}\right)}{m} \quad$ when $x=-l$,

where $l$ is the distance extended under the sea, $K_{3}$ is the hydraulic conductivity of the offshore confined aquifer, and $K^{\prime}$ and $m$ are the permeability and thickness of the outlet-capping, respectively. The continuity conditions of the hydraulic head and flux at $x=0$, respectively require

$$
\begin{gathered}
\lim _{x \uparrow 0} h_{2}(x, t)=\lim _{x \downarrow 0} h_{2}(x, t), \\
T_{3} \lim _{x \uparrow 0} \frac{\partial h_{2}(x, t)}{\partial x}=T_{2} \lim _{x \downarrow 0} \frac{\partial h_{2}(x, t)}{\partial x} .
\end{gathered}
$$

The boundary conditions for equations (1a) and (1b) on the inland side may respectively be expressed as:

$$
\begin{aligned}
& \lim _{x \rightarrow \infty} \frac{\partial h_{1}(x, t)}{\partial x}=0 \\
& \lim _{x \rightarrow \infty} \frac{\partial h_{2}(x, t)}{\partial x}=0
\end{aligned}
$$

which states that the slopes of the hydraulic head approach zero at the infinite boundary.

\section{Present solution and special cases}

Some normalized parameters used in Xia et al (2007) are also adopted hereinafter for the convenience of comparison. The tidal wave propagation parameter is defined as $a_{1}=\sqrt{\omega S_{1} / 2 T_{1}}$ for the unconfined aquifer, $a_{2}=\sqrt{\omega S_{2} / 2 T_{2}}$ for the inland confined aquifer, and $a_{3}=\sqrt{\omega S_{3} / 2 T_{3}}$ for the offshore confined aquifer. The dimensionless leakage is $u_{i}=L_{i} / \omega S_{2}$ for the inland aquitard and $u_{o}=L_{o} / \omega S_{3}$ for the offshore aquitard. The dimensionless leakance of outlet-capping is $u_{c}=$ $K^{\prime} /\left(a_{3} m K_{3}\right)$. In addition, the dimensionless storativity for inland aquifer is introduced as $S_{i}=$ $S_{1} / S_{2}$ and that for offshore aquifer as $S_{o}=S_{3} / S_{2}$, the dimensionless transmissivity for inland aquifer is defined as $T_{i}=T_{1} / T_{2}$ and that for offshore aquifer as $T_{o}=T_{3} / T_{2}$, and the dimensionless hydraulic diffusivity for inland aquifer is denoted as $D_{i}=T_{i} / S_{i}$ and that for offshore aquifer as $D_{o}=T_{o} / S_{o}$. The solutions of $h_{1}(x, t)$ and $h_{2}(x, t)$ for the inland unconfined and confined aquifers $(x>0)$ are, respectively, expressed as:

$$
\begin{aligned}
h_{1}(x, t)= & h_{\mathrm{MSL}}+\operatorname{Re}\left[A _ { 0 } \left(\alpha_{1} e^{-\lambda_{1} x}\right.\right. \\
& \left.\left.+\alpha_{2} e^{-\lambda_{2} x}\right) e^{-i \omega t}\right], \\
h_{2}(x, t)= & h_{\mathrm{MSL}}+\operatorname{Re}\left[A _ { 0 } \left(\alpha_{1} \beta_{1} e^{-\lambda_{1} x}\right.\right. \\
& \left.\left.+\alpha_{2} \beta_{2} e^{-\lambda_{2} x}\right) e^{-i \omega t}\right] .
\end{aligned}
$$

The solution for offshore aquifer $(x<0)$ is:

$$
\begin{aligned}
h_{2}(x, t)= & h_{\mathrm{MSL}}+\operatorname{Re}\left[A _ { 0 } \left(\alpha_{3} e^{\lambda_{3} x}\right.\right. \\
& \left.\left.+\alpha_{4} e^{-\lambda_{3} x}+\beta_{3}\right) e^{-i \omega t}\right]
\end{aligned}
$$

where Re denotes the real part of the complex expression and the variables $\alpha_{1}, \alpha_{2}, \alpha_{3}, \alpha_{4}, \beta_{1}, \beta_{2}$, $\beta_{3}, \lambda_{1}, \lambda_{2}$ and $\lambda_{3}$ are defined by equations (A6a-o), respectively, in Appendix A. Derivations of the other solutions considered as special cases of the present solution are given in Appendix B and the discussions of special cases are as follows:

\subsection{Ignoring water table fluctuations in upper unconfined aquifer}

If $T_{o} \rightarrow 1$ and $D_{i} \rightarrow 0$, the water table of the unconfined aquifer can then be considered as maintained constant. Accordingly, Xia et al's (2007) solution with neglecting the water table fluctuation is indeed a special case of the present solution. Xia et al (2007) mentioned that their solution 
can reduce to the head fluctuation solution of $\mathrm{Li}$ et al (2007) if the semi-permeable layer is replaced by an impermeable one. Therefore, the solution of Li et al (2007) is also a special case of the present solution. In addition, the solution of Geng et al (2009) is a special case of the present solution when the leakage of aquitard is zero.

\subsection{Ignoring outlet-capping effect in offshore aquifer}

When $m \rightarrow 0$, the thickness of the outlet-capping is very thin and negligible. The effect of outletcapping is negligible and the extended roof is under the free flow condition. Under this circumstance, the present solution is equal to the solution presented in Chuang and Yeh (2008). It is interesting to note that the solution of Li and Jiao (2001b) is a special case of the one presented in Chuang and Yeh (2008). Therefore, the solutions for the coastal confined aquifer with a roof extending over a certain distance under the tidal water, such as $\mathrm{Li}$ and Jiao (2001b) and Chuang and Yeh (2008), are all special cases of the present solution.

\subsection{Extending roof length of offshore aquifer to infinity}

If the roof length of the offshore aquifer extends to infinity, i.e., $l \rightarrow \infty$, the new solution (equations $3 \mathrm{a}-\mathrm{c}$ ), will reduce to the solution for head responses in a coupled coastal confined aquifer system consisting of a semi-permeable layer and a confined aquifer extending over an infinite distance under the sea. Then the present solution is equal to the one of Chuang and Yeh (2007). The solution for the leaky confined aquifer (i.e., equations $3 \mathrm{~b}$ and $3 \mathrm{c}$ ) should reduce to the solution presented in Van der Kamp (1972) when both leakages of the offshore and inland aquitards are equal to zero. Therefore, the solutions presented in Van der Kamp (1972) and Chuang and Yeh (2007) for confined aquifers extending over an infinite distance under the sea are also special cases of the newly developed solution.

\subsection{Ignoring both outlet-capping and roof length}

When the conditions $l \rightarrow 0$ and $m \rightarrow 0$ are held, the roof does not extend under the sea and the effect of outlet-capping on the head fluctuation in the confined aquifer is negligible. These two conditions are also applied to Jeng et al (2002). Equations (3a) and (3b) are exactly the same as the ones of Jeng et al (2002, equations 12 and 13) except that the variables of $\beta_{1}$ and $\beta_{2}$ are in terms of dimensionless parameters. Jeng et al (2002) demonstrated that their solution reduces to that of Jiao and Tang (1999) when the water table is uniform. Jiao and Tang (1999) mentioned that their solution is the same as that given by Ferris (1951) if there is no leakage from the above unconfined aquifer. In addition, Li and Jiao (2001b) showed that the solution of confined aquifer is equal to the one developed by Jacob (1950) when both the leakage term and roof length are zero. Obviously, those solutions of Jacob (1950), Ferris (1951), Jiao and Tang (1999), and Jeng et al (2002) for the tidal confined aquifer with zero offshore length can also be considered as our special cases.

\section{Results and discussion for present solution}

Equations $(3 \mathrm{a}-\mathrm{c})$ are the solutions for the groundwater heads in the inland unconfined aquifer, inland confined aquifer, and offshore part of the confined aquifer, respectively. Since most field studies on coastal aquifer systems focus on the inland part of aquifer and the observation of groundwater heads in the offshore aquifer is usually unavailable. Thus, only the groundwater heads in inland part of the aquifers are addressed herein (Li and Jiao 2001b). Some lumped parameters are introduced for convenience of discussion. The amplitude coefficient $\left(C_{e}\right)$ is defined as $C_{e}=$ $\operatorname{Re}\left[\alpha_{1} \beta_{1}+\alpha_{2} \beta_{2}\right]$ to represent the maximum amplitude of groundwater fluctuation. In addition, the constant phase shift $(\varphi)$ of the inland confined aquifer is defined as $\varphi=\operatorname{Re}\left[\alpha_{1} \beta_{1}+\alpha_{2} \beta_{2}\right] /$ $\operatorname{Im}\left[\alpha_{1} \beta_{1}+\alpha_{2} \beta_{2}\right]$, where Im represents the imaginary part of the complex expression, to represent the phase angle when the groundwater fluctuation has the maximum amplitude. Reasonable ranges of aquifer parameter values reported in the literature (e.g., Li et al 2001b; Jeng et al 2002) are listed in table 1 for the case study. The formation materials of the offshore confined aquifer and its outlet-capping are considered to be fine sand and silt, respectively, in this study. The thickness of the outlet-capping is assumed to be $10 \mathrm{~m}$. Thus, the hydraulic conductivity of fine sand formation ranges from $2 \times 10^{-2}$ to $20 \mathrm{~m} /$ day and that of silt formation ranges from $8 \times 10^{-5}$ to $2 \mathrm{~m} /$ day (Domenico and Schwartz 1997). In addition, the values of the tidal wave propagation parameter $a_{2}$ and $a_{3}$ are in the range of $10^{-1} \sim 10^{-3} \mathrm{~m}^{-1}$ ( $\mathrm{Li}$ and Jiao 2001b). The leakance of outletcapping, $u_{c}=K^{\prime} /\left(a_{3} m K_{3}\right)$, may therefore fall in the range from $4 \times 10^{-6}$ to $10^{4}$. Accordingly, the consideration for the value of the logarithm of the leakance of outlet-capping, $\lg u_{c}$, varied from -3 to 3 seems to be reasonable for coastal aquifers. The 
Table 1. Input data for the case study (Li et al 2001b; Jeng et al 2002).

\begin{tabular}{ll}
\hline Parameter & \multicolumn{1}{c}{ Value } \\
\hline Amplitude of tide $A_{0}$ & $0.65 \mathrm{~m}$ \\
Mean sea level $h_{\mathrm{MSL}}$ & $0 \mathrm{~m}$ \\
Out-capping thickness $m$ & 0 or varying $\mathrm{m}$ \\
Specific yield $S_{1}$ & $0.1 \sim 10^{-3}$ \\
Storativity of inland leaky confined aquifer $S_{2}$ & $10^{-3} \sim 10^{-5}$ \\
Storativity of offshore leaky confined aquifer $S_{3}$ & $10^{-3} \sim 10^{-5}$ \\
Tidal frequency $\omega$ & $2 \pi \mathrm{rad} /$ day \\
Tidal propagation parameter of aquifer $a_{1} \sim 3$ & $10^{-1} \sim 10^{-3} / \mathrm{m}$ \\
Transmissivity of unconfined aquifer $T_{1}$ & $2000 \mathrm{~m}^{2} / \mathrm{day}$ or varying \\
Transmissivity of inland leaky confined aquifer $T_{2}$ & $2000 \mathrm{~m}^{2} / \mathrm{day}$ or varying \\
Transmissivity of offshore leaky confined aquifer $T_{3}$ & $2000 \mathrm{~m}^{2} / \mathrm{day}$ or varying \\
Dimensionless inland leakage $u_{i}=L_{i} / \omega S_{2}$ & $0.1 \mathrm{or} 10$ \\
Dimensionless offshore leakage $u_{o}=L_{o} / \omega S_{3}$ & 0.1 or 10 \\
Dimensionless out-capping leakage $u_{c}=K^{\prime} /\left(a_{3} m K\right)$ & 0 or varying \\
Dimensionless tidal propagation parameter $a_{3} l$ & 0 or varying $(l=$ roof length $)$ \\
Dimensionless inland storativity $S_{i}=S_{1} / S_{2}$ & 1 or $10^{4}$ \\
Dimensionless offshore storativity $S_{o}=S_{3} / S_{2}$ & $10^{-2} \sim 10^{2}$ \\
Dimensionless inland transmissivity $T_{i}=T_{1} / T_{2}$ & 1 or varying \\
Dimensionless offshore transmissivity, $T_{o}=T_{3} / T_{2}$ & 1 or varying \\
Dimensionless inland hydraulic diffusivity $D_{i}=T_{i} / S_{i}$ & $10^{-4}$ or 1 or varying \\
Dimensionless offshore hydraulic diffusivity $D_{o}=T_{o} / S_{o}$ & $10^{-2}$ or 1 or varying \\
\hline
\end{tabular}

effects of dimensionless out-capping, aquitard leakages and hydraulic diffusivities on the groundwater heads in the inland confined aquifer are addressed in the following sections.

\subsection{The effect of dimensionless inland leakage on head fluctuation}

Figure 2 shows the curves of the amplitude coefficient $C_{e}$ and phase shift $\varphi$ versus the logarithm of dimensionless outlet-capping leakage $\lg u_{c}$ when the dimensionless inland leakage $u_{i}$ varies from 0.1 to 10 with parameters $a_{3} l=0.1, T_{e}=0.5, D_{o}=1$ and $u_{o}=1$. The figure indicates that both $C_{e}$ and $\varphi$ are close to constant when $\lg u_{c}<-3$ or $>3$ and the behaviours of $C_{e}$ and $\varphi$ are therefore not discussed in these two regions. The solid lines denote the present solution with $D_{i}=0.1$ while the dashed lines stand for the solution with $D_{i}=0$ implying that the water table fluctuation in the unconfined aquifer is negligible. The solid lines in the figure display that $C_{e}$ increases with $u_{c}$ for all $u_{i}$ in the range $-1<\lg u_{c}<2$. In addition, the solid lines also indicate that the influence of $u_{i}$ is large for the groundwater heads in the inland confined aquifer and the value of $C_{e}$ increases with $u_{i}$ when $u_{c}$ is relatively small (say $\lg u_{c}<0.5$ ). On the contrary, the influence of $u_{i}$ is relatively small for the groundwater head and $C_{e}$ decreases with increasing $u_{i}$ when $u_{c}$ is large $\left(\lg u_{c}>0.5\right)$. The dashed lines of figure $2(\mathrm{a})$ display that the $C_{e}$ decreases with increasing $u_{i}$ for all $u_{c}$. The influence of water table fluctuation on the groundwater head in the inland confined aquifer increases significantly with $u_{i}$ for all $u_{c}$ and decreases with increasing $u_{c}$ for all $u_{i}$ as compared the solid and dashed lines shown in figure 2(a). Both solid and dashed lines in figure 2(b) show that the influence of $u_{i}$ on $\varphi$ decreases significantly with increasing $u_{c}$. Moreover, the solid lines display that $\varphi$ decreases with increasing $u_{c}$ when $u_{i} \geq 1$. However, $\varphi$ initially increases with $u_{c}$, reaches a peak value at $\lg u_{c}=-0.5$, and then decreases as $u_{c}$ increases for the case of $u_{i}=0.1$. The dashed lines in figure 2(b) show that the $\varphi$ has a peak value near $\lg u_{c}=0.5$ and decreases when away from the peak value when $u_{i}=0.1$ or 1 , but the $\varphi$ increases with increasing $u_{c}$ when $u_{i}=10$. This phenomenon is caused by the combined effects of the outlet-capping and aquitard leakages. The dashed lines also display that $\varphi$ increases with decreasing $u_{i}$ for all $u_{c}$. In addition, the value of $\varphi$ depends on the water table fluctuation in the unconfined aquifer when $u_{c}$ is relatively small and the effect of the water table fluctuation is relatively small when $u_{c}$ is large as demonstrated in figure $2(\mathrm{~b})$.

\subsection{The effect of dimensionless offshore leakage on head fluctuation}

Figure 3 displays the curves of the $C_{e}$ and $\varphi$ versus $\lg u_{c}$ denoted by the solid lines for $D_{i}=0.1$ and the 


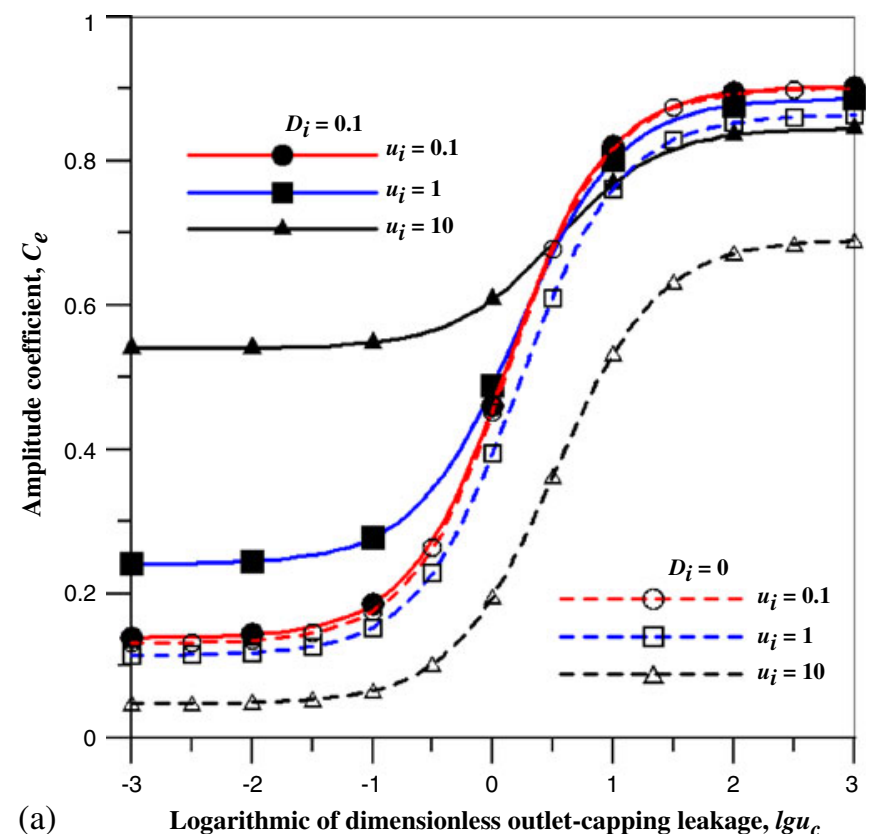

(a) Logarithmic of dimensionless outlet-capping leakage, $\lg u_{c}$

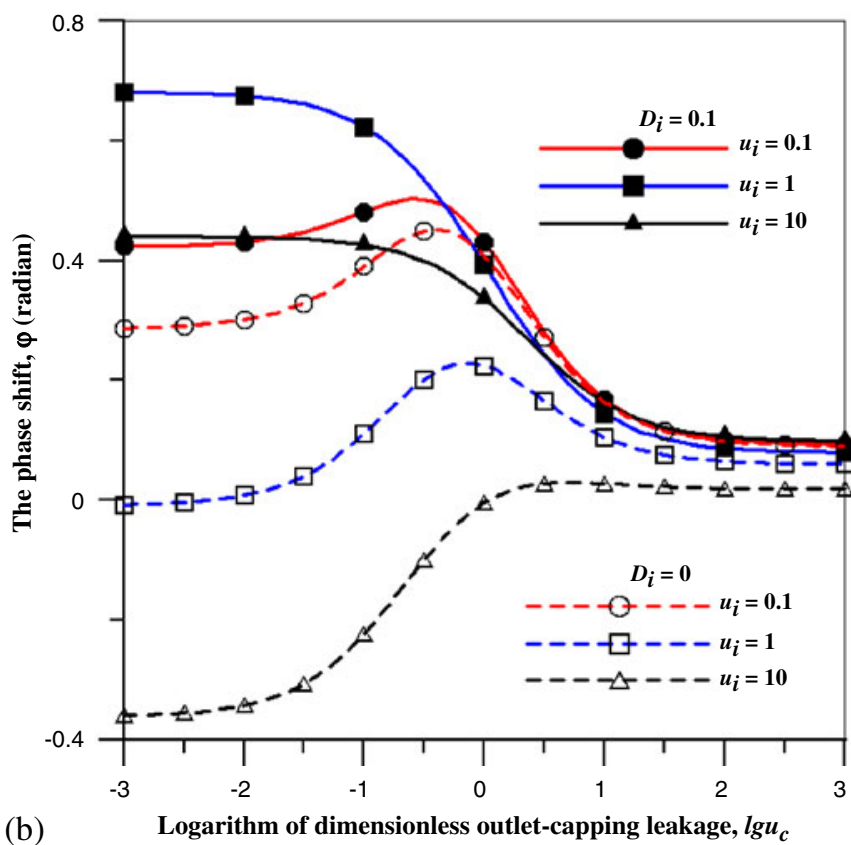

Figure 2. The curves for (a) amplitude coefficient $\left(C_{e}\right)$ and (b) the phase shift $(\varphi)$ versus logarithm of dimensionless outlet-capping leakage $\left(\lg u_{c}\right)$ when the dimensionless inland leakage $\left(u_{i}\right)$ varies from 0.1 to 10 with parameters $a_{3} l=0.1$, $T_{e}=0.5, D_{i}=0$ or $0.1, D_{o}=1$ and $u_{o}=1$.

dashed line for $D_{i}=0$ when the $u_{o}$ varies from 0.1 to 10 with parameters $a_{3} l=0.1, T_{e}=0.5, D_{o}=1$ and $u_{i}=1$. Both figures $3(\mathrm{a})$ and $3(\mathrm{~b})$ indicate that the influence of $u_{o}$ on the $C_{e}$ and $\varphi$ decreases gradually with increasing $u_{c}$. The dynamic effect of water table fluctuation on the head response in the confined aquifer increases with decreasing $u_{c}$ for all $u_{o}$. Figure $3(\mathrm{a})$ shows that the $C_{e}$ increases with $u_{c}$ for all $u_{o}$. In addition, the influence of $u_{c}$ on $C_{e}$

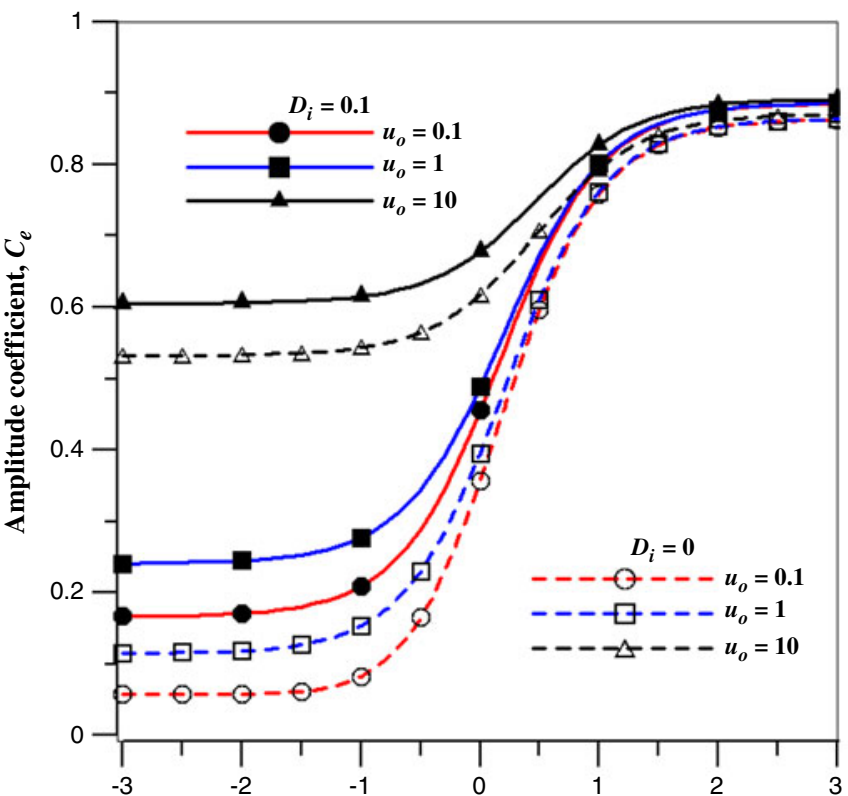

(a) Logarithm of dimensionless outlet-capping leakage, $\lg u_{c}$

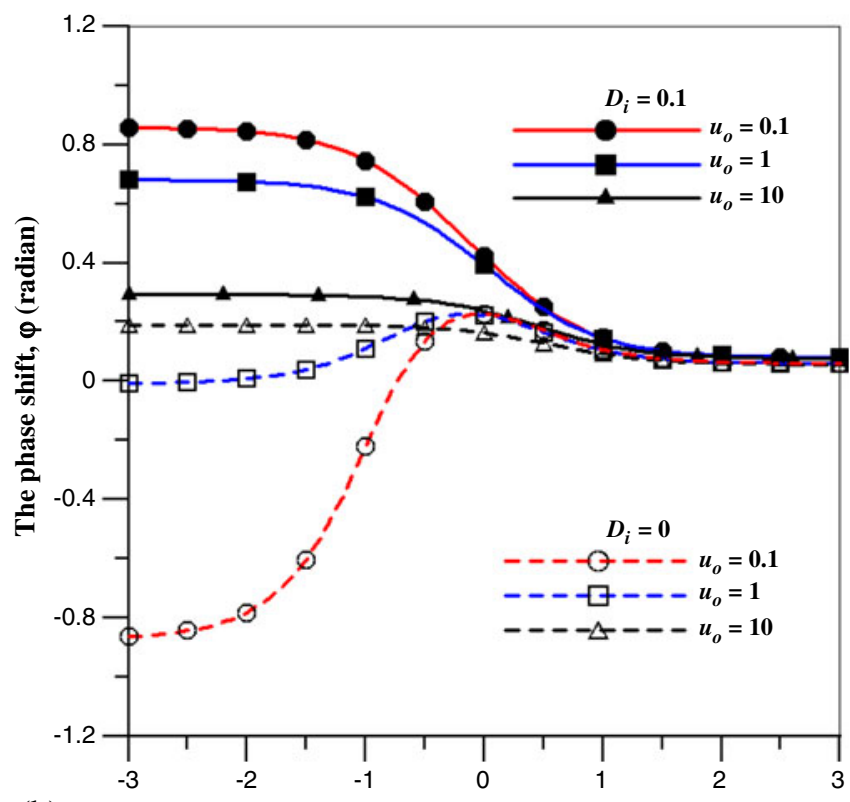

(b)

Logarithm of dimensionless outlet-capping leakage, $\lg u_{c}$

Figure 3. The curves for (a) amplitude coefficient $\left(C_{e}\right)$ and (b) the phase shift $(\varphi)$ versus logarithm of dimensionless outlet-capping leakage $\left(\lg u_{c}\right)$ when the dimensionless offshore leakage $\left(u_{o}\right)$ varies from 0.1 to 10 with parameters $a_{3} l=0.1, T_{e}=0.5, D_{i}=0$ or $0.1, D_{o}=1$ and $u_{i}=1$.

is large and this influence decreases as $u_{o}$ increases when $-1<\lg u_{c}<1$. These phenomena reflect the joint effects of $u_{c}$ and $u_{o}$ on the groundwater heads in inland part of the aquifer are very significant. The influence of $u_{o}$ is large when $u_{c}$ is small; on the other hand, the influence of $u_{o}$ is small when $u_{c}$ is large. The solid lines in figure $3(\mathrm{~b})$ exhibit that the $\varphi$ decreases with increasing $u_{c}$. The influence of $u_{c}$ on $\varphi$ decreases with increasing $u_{o}$ when 
$-2<\lg u_{c}<1$. The dashed lines display that the $\varphi$ has a peak value near $\lg u_{c}=0$ and decreases when away from the peak value indicating that the effect of $u_{c}$ on $\varphi$ is large when $u_{o}=0.1$ or 1 , but the $\varphi$ decreases slightly with increasing $u_{c}$ when $u_{o}=10$. On the other hand, the effect of $u_{c}$ on $\varphi$ is almost negligible when $\lg u_{c}>1$. Obviously, the effect of $u_{o}$ on $\varphi$ is small when $u_{c}$ is large as demonstrated in the figure.

\subsection{The simultaneous effect of dimensionless leakages on head fluctuation}

If the dimensionless inland leakage $\left(u_{i}\right)$ and offshore leakage $\left(u_{o}\right)$ are set equal, then the dimensionless leakage $u$ is $u=u_{i}=u_{o}$. The curves of $C_{e}$ and $\varphi$ versus the logarithm of the dimensionless out-capping leakage, $\lg u_{c}$, in the range between -3 and 3 are demonstrated in figure 4 for $u_{i}=u_{o}=u=1$ and 10, $a_{3} l=0.1, T_{e}=0.5$, $D_{i}=0.1$, and $D_{o}=1$. In figure 4 , the solid lines denote the present solution while the dashed lines represent the solution of Xia et al (2007) in which the water table fluctuation is not considered. Figure 4(a) shows that $C_{e}$ increases with $u_{c}$ for all $u$. However, the $C_{e}$ predicted by the present solution is obviously greater than that of Xia et al's (2007) solution for all $u_{c}$ and their difference increases with $u$ and decreases with increasing $u_{c}$. In other words, the $C_{e}$ will be underestimated if the water table fluctuation in the unconfined aquifer is neglected. Figure 4(b) displays a similar result that $\varphi$ in the present solution is obviously greater than that of Xia et al's (2007) solution for all $u_{c}$. The $\varphi$ decreases with increasing $u_{c}$ in the present solution and the influence of $u_{c}$ on $\varphi$ increases as $u$ decreases. The effects of $u_{c}$ on $\varphi$ predicted by the present solution and Xia et al's (2007) solution are very different. In figure 4(b), the dashed lines display that the $\varphi$ increases slightly with $u_{c}$ when $\lg u_{c}<0.5$ and decreases slightly with increasing $u_{c}$ when $\lg u_{c}>0.5$ for $u=10$. On the other hand, the $\varphi$ increases with $u_{c}$ when $\lg u_{c}<0$ and decreases with increasing $u_{c}$ when $\lg u_{c}>0$ for $u=1$. Figure 4 indicates that the influence of the water table fluctuation in the unconfined aquifer on the $C_{e}$ and $\varphi$ when $\lg u_{c}=-3$ is obviously larger than that when $\lg u_{c}=3$. Note that the outletcapping might be considered as no-flow boundary when $\lg u_{c}=-3$ while the capping is considered as free-flow boundary when $\lg u_{c}=3$. The predicted results from Xia et al's (2007) solution are significantly different from those of the present solution. The use of Xia et al's (2007) solution should therefore be cautious if the water table fluctuation is significant.

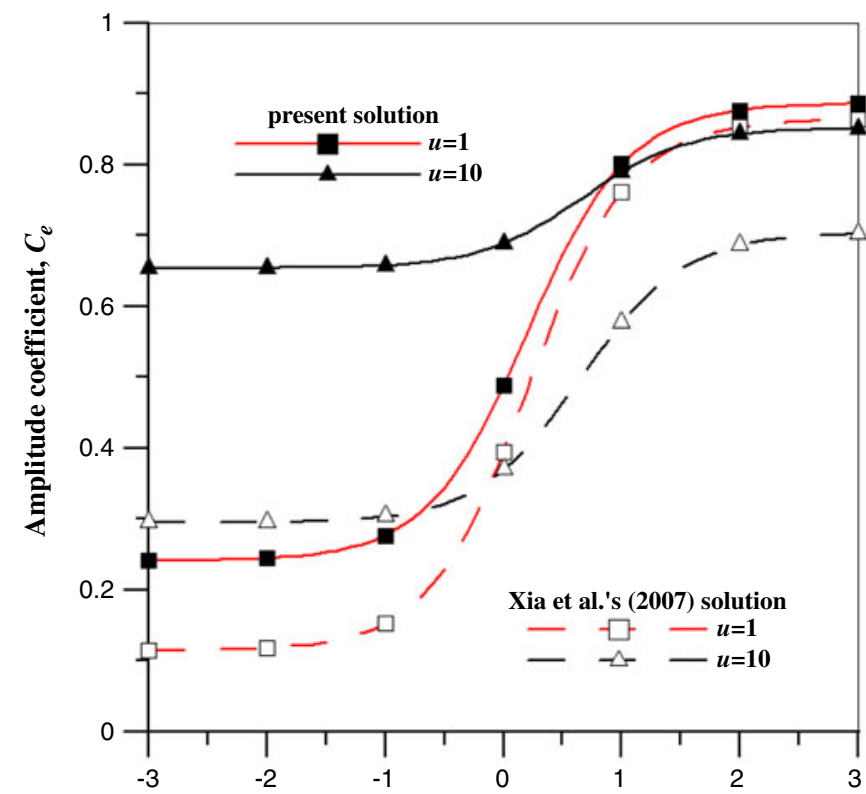

(a) Logarithm of dimensionless outlet-capping leakage, $\lg u_{c}$

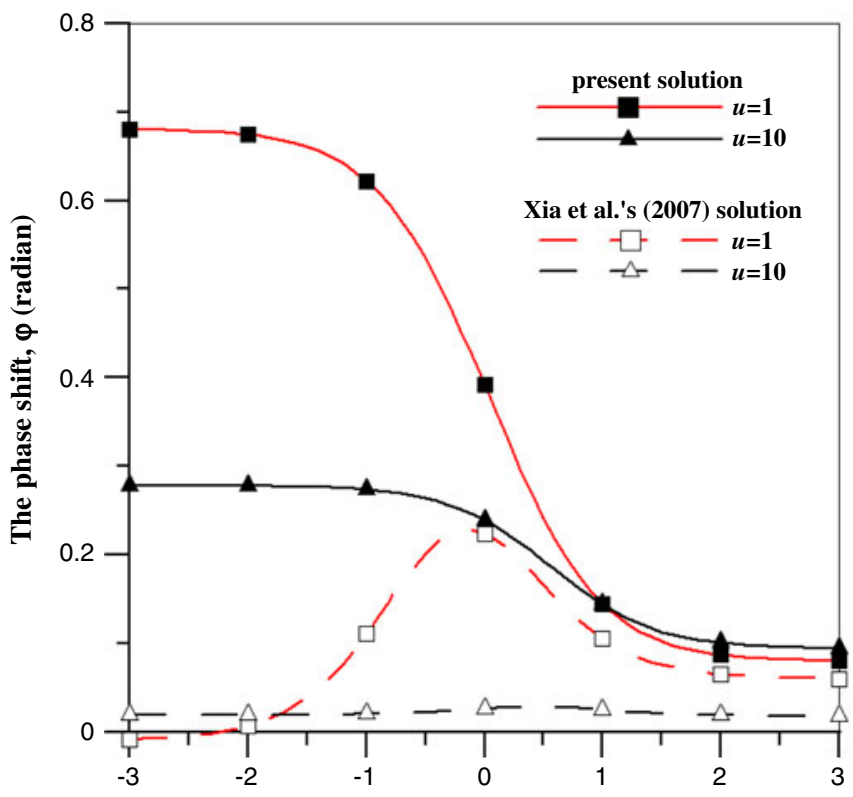

(b) Logarithm of dimensionless outlet-capping leakage, $\lg u_{c}$

Figure 4. The curves for (a) amplitude coefficient $\left(C_{e}\right)$ and (b) the phase shift $(\varphi)$ versus logarithm of dimensionless outlet-capping leakage $\left(\lg u_{c}\right)$ when the dimensionless leakage $\left(u=u_{i}=u_{o}\right)$ is equal to 1 and 10 with parameters $a_{3} l=0.1, T_{e}=0.5, D_{i}=0.1$, and $D_{o}=1$.

\subsection{The effects of dimensionless hydraulic diffusivities on head fluctuation}

Figure 5 shows that the curves of $C_{e}$ and $\varphi$ versus $\lg u_{c}$ when $D_{i}$ varies from 0 to 1 with parameters $a_{3} l=0.1, D_{o}=1, T_{e}=0.5, u_{i}=1$ and $u_{o}=1$. Figure 5(a) displays that the $C_{e}$ increases dramatically with $u_{c}$ when $-1<\lg u_{c}<1$ for all $D_{i}$. In addition, a larger $D_{i}$ gives a larger $C_{e}$. In other 

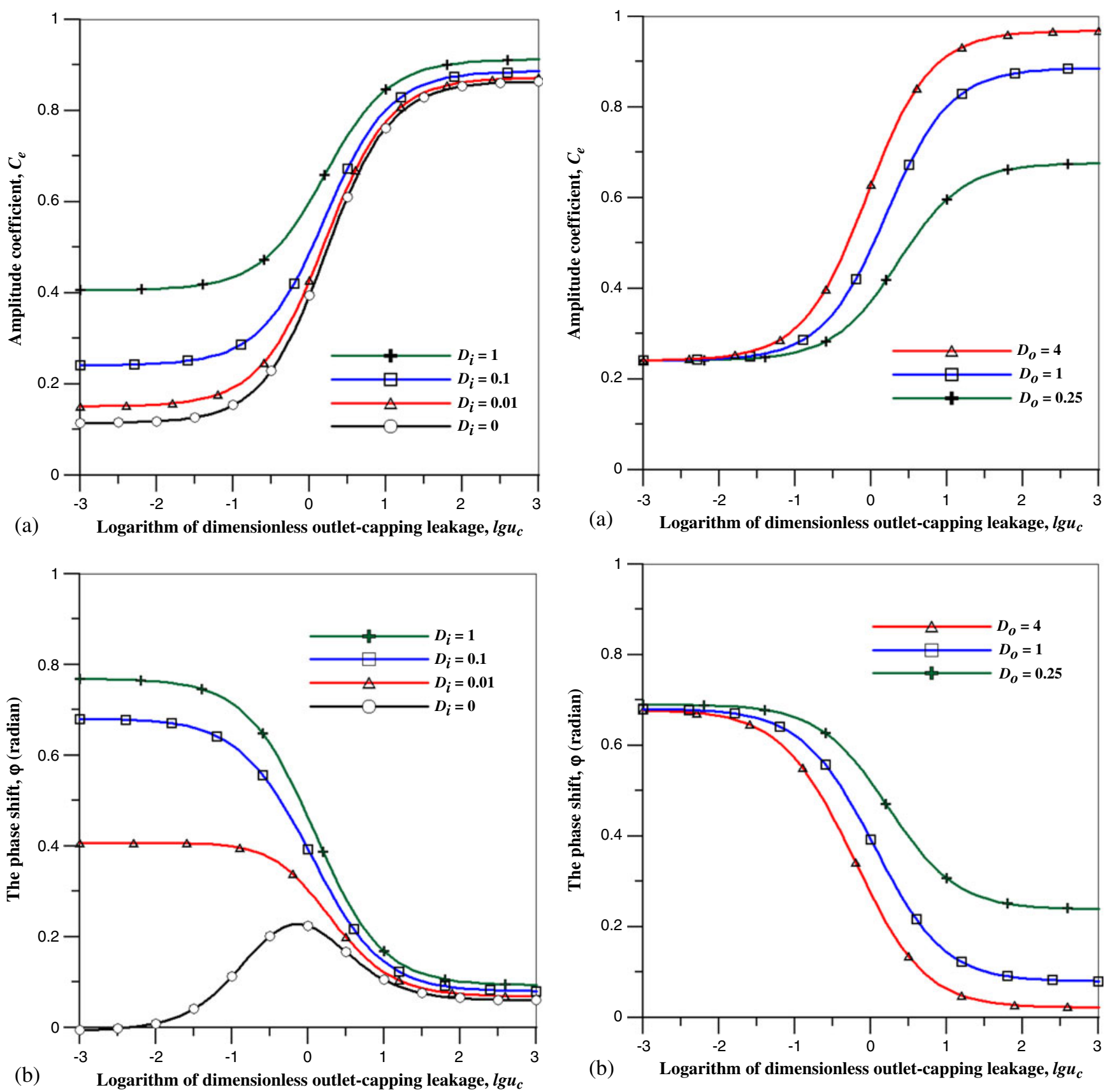

Figure 5. The curves for (a) amplitude coefficient $\left(C_{e}\right)$ and (b) the phase shift $(\varphi)$ versus logarithm of dimensionless outlet-capping leakage $\left(\mathrm{lg} u_{c}\right)$ when the dimensionless hydraulic diffusivity $\left(D_{i}\right)$ varies from 0 to 1 with parameters $a_{3} l=0.1, D_{o}=1, T_{e}=0.5, u_{i}=1$ and $u_{o}=1$.

words, the effect of $D_{i}$ is large when $u_{c}$ is relatively small. By contrast, the influence of $D_{i}$ is small when $u_{c}$ is relatively large. The figure also shows that the discrepancy caused by neglecting the water table fluctuation in the unconfined aquifer increases with $D_{i}$ and decreases with increasing $u_{c}$. Figure $5(\mathrm{~b})$ shows that the $\varphi$ decreases as $u_{c}$ increases for $D_{i}>0.01$ and the decrease of $\varphi$ is very rapid for $\lg u_{c}$ in the range from -1 to 1 . The figure also shows that the $\varphi$ has a peak value near

Figure 6. The curves for (a) amplitude coefficient $\left(C_{e}\right)$ and (b) the phase shift $(\varphi)$ versus logarithm of dimensionless outlet-capping leakage $\left(\lg u_{c}\right)$ when the dimensionless hydraulic diffusivity $\left(D_{o}\right)$ varies from 0.25 to 4 with parameters $a_{3} l=0.1, D_{i}=0.1, T_{e}=0.5, u_{i}=1$ and $u_{o}=1$.

$\lg u_{c}=0$ when $D_{i}=0$ and the effect of $D_{i}$ on $\varphi$ in the range of $\lg u_{c}<0$ is larger than that in the range of $\lg u_{c}>0$.

Figure 6 displays the curves for $C_{e}$ and $\varphi$ versus logarithm of dimensionless outlet-capping leakage when $D_{o}$ varies from 0.25 to 4 with parameters $a_{3} l=0.1, D_{i}=0.1, T_{e}=0.5, u_{i}=1$ and $u_{o}=1$. Figure 6(a) shows that the $C_{e}$ increases with $u_{c}$ for all $D_{o}$ and the influence of $u_{c}$ on $C_{e}$ increases with $D_{o}$. The figure also shows that the effect of 
$D_{o}$ increases with $u_{c}$ for $C_{e}$. Figure $6(\mathrm{~b})$ displays that the $\varphi$ decreases with increasing $u_{c}$ for all $D_{o}$ and the influence of $u_{c}$ on $\varphi$ increases with $D_{o}$. In addition, the figure also displays the effect of $D_{o}$ on $\varphi$ increases with $u_{c}$.

\section{Conclusion}

A new analytical solution has been developed for a coastal aquifer system consisting of an unconfined aquifer, an aquitard, and a leaky confined aquifer. The unconfined aquifer ends at the coast while the aquitard and confined aquifer extend over a finite distance under the sea and terminate with an outlet-capping. The solution has been demonstrated to be a generalization of most existing analytical solutions for various types of coastal aquifer systems. In addition this solution can be used to explore the influences of the outlet-capping leakage, the hydraulic diffusivities, and the leakages of the inland and offshore aquitards on the head response in the leaky confined aquifer. It is found that the interaction between the outletcapping leakage and the dynamic effect of water table fluctuation on the head response in the leaky confined aquifer is very significant for the aquifer with an outlet-capping in a coupled coastal aquifer system.

\section{Acknowledgements}

This research was partially supported by Taiwan National Science Council under the grants NSC 992221-E-009-062-MY3, NSC 100-2221-E-009-106, and NSC100-3113-E-007-011. The authors would also like to thank the three anonymous reviewers for their valuable and constructive comments.

\section{Appendix A. Derivation of solutions to equations $(1 \mathrm{a}-\mathrm{c})$ subject to equations $(2 \mathrm{a}-\mathrm{f})$}

Let $H_{1}(x, t)$ and $H_{2}(x, t)$ be complex functions of the real variables $x$ and $t$ that satisfy the governing equations $(1 \mathrm{a}-\mathrm{c})$ and the conditions $(2 \mathrm{a}-\mathrm{f})$. Assume that the solutions to equations $(1 \mathrm{a}-\mathrm{c})$ subject to equations $(2 \mathrm{a}-\mathrm{f})$ are $h_{1}(x, t)$ and $h_{2}(x, t)$ and expressed, respectively, as:

$$
\begin{aligned}
& h_{1}(x, t)=h_{\mathrm{MSL}}+\operatorname{Re}\left[H_{1}(x, t)\right] \\
& h_{2}(x, t)=h_{\mathrm{MSL}}+\operatorname{Re}\left[H_{2}(x, t)\right]
\end{aligned}
$$

where Re denotes the real part of the complex expression. Consider that

$$
\begin{aligned}
& H_{1}(x, t)=A_{0} X_{1}(x) e^{-i \omega t} \\
& H_{2}(x, t)=A_{0} X_{2}(x) e^{-i \omega t}
\end{aligned}
$$

where $X_{1}(x)$ and $X_{2}(x)$ are unknown functions of $x$ and $i=\sqrt{-1}$. Substituting equations (A2a) and (A2b) into following nine equations $(1 \mathrm{a}-\mathrm{c})$ and $(2 \mathrm{a}-$ f), which $H_{1}(x, t)$ and $H_{2}(x, t)$ satisfy, and dividing the results by $A_{0} e^{-i \omega t}$ yield the results for the inland aquifers $(x>0)$ as:

$$
\begin{array}{r}
X_{1}^{\prime \prime}(x)+2 a_{1}^{2}\left(i-\frac{u_{i}}{S_{i}}\right) X_{1}(x) \\
+\frac{2 a_{1}^{2} u_{i}}{S_{i}} X_{2}(x)=0 \\
X_{2}^{\prime \prime}(x)+2 a_{2}^{2}\left(i-u_{i}\right) X_{2}(x) \\
+2 a_{2}^{2} u_{i} X_{1}(x)=0
\end{array}
$$

and the result for the offshore aquifer $(x<0)$ as:

$$
X_{2}^{\prime \prime}(x)+2 a_{3}^{2}\left(i-u_{o}\right) X_{2}(x)=2 a_{3}^{2}\left(T_{e} i-u_{o}\right) .
$$

The tidal boundaries, equations (4a) and (4b) may be respectively written as:

$$
\begin{gathered}
X_{1}(0)=1, \\
X_{2}^{\prime}(-l)-a_{3} u_{c} X_{2}(-l)+a_{3} u_{c}=0 .
\end{gathered}
$$

In addition, the continuity conditions of equations (4c) and (4d) may be respectively expressed as:

$$
\begin{gathered}
\lim _{x \uparrow 0} X_{2}(x)=\lim _{x \downarrow 0} X_{2}(x), \\
T_{o} \lim _{x \uparrow 0} X_{2}^{\prime}(x)=\lim _{x \downarrow 0} X_{2}^{\prime}(x) .
\end{gathered}
$$

The boundary conditions, equations (4e-f) may also be respectively written as:

$$
\begin{aligned}
& X_{1}^{\prime}(+\infty)=0, \\
& X_{2}^{\prime}(+\infty)=0 .
\end{aligned}
$$

Thus, the general solutions to equations (A3a-c) for inland aquifers $(x>0)$ are:

$$
\begin{gathered}
X_{1}(x)=\alpha_{1} e^{-\lambda_{1} x}+\alpha_{2} e^{-\lambda_{2} x} \\
X_{2}(x)=\alpha_{1} \beta_{1} e^{-\lambda_{1} x}+\alpha_{2} \beta_{2} e^{-\lambda_{2} x}
\end{gathered}
$$


and for offshore aquifer $(x<0)$ is:

$$
X_{2}(x)=\alpha_{3} e^{\lambda_{3} x}+\alpha_{4} e^{-\lambda_{3} x}+\beta_{3}
$$

where variables $\alpha_{1}, \alpha_{2}, \alpha_{3}, \beta_{1}, \beta_{2}, \beta_{3}, \lambda_{1}, \lambda_{2}$, and $\lambda_{3}$ are defined, respectively as:

$$
\begin{array}{cl}
\alpha_{1}=\frac{D_{1}}{D} & (\mathrm{~A} 6 \mathrm{a}) \\
\alpha_{2}=\frac{D_{2}}{D} & (\mathrm{~A} 6 \mathrm{~b}) \\
\alpha_{3}=\frac{D_{3}}{D} & (\mathrm{~A} 6 \mathrm{c}) \\
\alpha_{4}=\frac{D_{4}}{D} & (\mathrm{~A} 6 \mathrm{~d}) \\
\times\left(\lambda_{3}-a_{3} u_{c}\right) e^{-\lambda_{3} l} & \left(T_{o} \beta_{1} \lambda_{3}-T_{o} \beta_{2} \lambda_{3}-\beta_{1} \lambda_{1}+\beta_{2} \lambda_{2}\right) \\
+ & \left(\lambda_{3}+a_{3} u_{c}\right) e^{\lambda_{3} l} \\
\times & \left(-T_{o} \beta_{1} \lambda_{3}+T_{o} \beta_{2} \lambda_{3}-\beta_{1} \lambda_{1}+\beta_{2} \lambda_{2}\right)(\mathrm{A} 6 \mathrm{e}) \\
D_{1}= & \left(\lambda_{3}-a_{3} u_{c}\right) e^{-\lambda_{3} l} \\
& \times\left(-T_{o} \beta_{2} \lambda_{3}+T_{o} \beta_{3} \lambda_{3}+\beta_{2} \lambda_{2}\right) \\
& +\left(\lambda_{3}+a_{3} u_{c}\right) e^{\lambda_{3} l} \\
& \times\left(T_{o} \beta_{2} \lambda_{3}-T_{o} \beta_{3} \lambda_{3}+\beta_{2} \lambda_{2}\right) \\
& -2 \lambda_{3} a_{3} u_{c} T_{o}\left(1-\beta_{3}\right) \\
& +\left(\lambda_{3}+a_{3} u_{c}\right) e^{\lambda_{3} l} \\
D_{2}= & \left(\lambda_{3}-a_{3} u_{c}\right) e^{-\lambda_{3} l} \\
& \times\left(T_{o} \beta_{1} \lambda_{3}-T_{o} \beta_{3} \lambda_{3}-\beta_{1} \lambda_{1}\right) \\
&
\end{array}
$$

$$
\begin{aligned}
D_{3}= & \left(\lambda_{3}+a_{3} u_{c}\right) e^{\lambda_{3} l} \\
& \times\left(-\beta_{1} \beta_{2} \lambda_{1}+\beta_{1} \beta_{3} \lambda_{1}+\beta_{1} \beta_{2} \lambda_{2}-\beta_{2} \beta_{3} \lambda_{2}\right) \\
& -a_{3} u_{c}\left(1-\beta_{3}\right) \\
& \times\left(T_{o} \beta_{1} \lambda_{3}-T_{o} \beta_{2} \lambda_{3}-\beta_{1} \lambda_{1}+\beta_{2} \lambda_{2}\right)(\mathrm{A} 6 \mathrm{~h}) \\
D_{4}= & \left(\lambda_{3}-a_{3} u_{c}\right) e^{-\lambda_{3} l} \\
& \times\left(-\beta_{1} \beta_{2} \lambda_{1}+\beta_{1} \beta_{3} \lambda_{1}+\beta_{1} \beta_{2} \lambda_{2}-\beta_{2} \beta_{3} \lambda_{2}\right) \\
- & a_{3} u_{c}\left(1-\beta_{3}\right) \\
& \times\left(T_{o} \beta_{1} \lambda_{3}-T_{o} \beta_{2} \lambda_{3}+\beta_{1} \lambda_{1}-\beta_{2} \lambda_{2}\right) \quad(\mathrm{A} 6 \mathrm{i}) \\
\beta_{1}=1-\frac{S_{i} B_{1}}{2 a_{1}^{2} u_{i}}-\frac{S_{i} i}{u_{i}} & (\mathrm{~A} 6 \mathrm{j}) \\
\beta_{2}=1-\frac{S_{i} B_{2}}{2 a_{1}^{2} u_{i}}-\frac{S_{i} i}{u_{i}} & (\mathrm{~A} 6 \mathrm{k}) \\
\beta_{3}=\frac{T_{e} i-u_{o}}{i-u_{o}} & (\mathrm{~A} 6 \mathrm{l}) \\
\lambda_{1}=\sqrt{B_{1}} & (\mathrm{~A} 6 \mathrm{~m}) \\
\lambda_{2}=\sqrt{B_{2}} &
\end{aligned}
$$

with variables $B_{1}$ and $B_{2}$ respectively defined as:

$$
\begin{aligned}
& B_{1}=-c_{1}-\sqrt{c_{1}^{2}-c_{2}} \\
& B_{2}=-c_{1}+\sqrt{c_{1}^{2}-c_{2}}
\end{aligned}
$$

and variables $c_{1}$ and $c_{2}$ respectively defined as:

$$
\begin{gathered}
c_{1}=-\left(a_{1}^{2} / S_{i}+a_{2}^{2}\right) u_{i}+\left(a_{1}^{2}+a_{2}^{2}\right) i, \\
c_{2}=-4 a_{1}^{2} a_{2}^{2}\left(1+u_{i} i / S_{i}+u_{i} i\right) .
\end{gathered}
$$

\section{Appendix B.}

\section{Derivation of other solutions considered as special cases}

\section{B1. Ignoring water table fluctuations in upper unconfined aquifer}

If $T_{o} \rightarrow 1, D_{i} \rightarrow 0$ and $u=u_{i}=u_{o}$, the variables $\alpha_{3}$ and $\alpha_{4}$ become $\alpha_{3 a}$ and $\alpha_{4 a}$, respectively. Based on equations $(\mathrm{A} 6 \mathrm{a}-\mathrm{s})$, one can obtain

$$
\begin{aligned}
\lambda_{1} & =\lambda_{2}=\lambda_{3} a_{2}(2 u-2 i)^{0.5} \\
& =a(p-q i)
\end{aligned}
$$

$$
\begin{gathered}
\beta_{3}=\frac{u-i T_{e}}{u-i}=\lambda-i \mu \\
X_{2}(x)=-\left(\alpha_{3 a} e^{\lambda_{3} x}+\alpha_{4 a} e^{-\lambda_{3} x}+\beta_{3}\right)
\end{gathered}
$$

for offshore aquifer $(-l<x<0)$ and

$$
\begin{aligned}
X_{2}(x) & =-\left(\alpha_{1} \beta_{1}+\alpha_{2} \beta_{2}\right) e^{-\lambda_{1} x} \\
& =-\gamma e^{-\lambda_{1} x}
\end{aligned}
$$


for inland aquifer $(x>0)$ with the variables $\gamma, \alpha_{3 a}$ and $\alpha_{4 a}$ defined, respectively as:

$$
\begin{aligned}
\alpha_{4 a}= & \frac{u_{c}\left(1-\beta_{3}\right)}{\left(u_{c}+(p-i q)\right)} e^{-\lambda_{3} l} \\
+ & \frac{\beta_{3}\left(u_{c}-(p-i q)\right)}{2\left(u_{c}+(p-i q)\right)} e^{-2 \lambda_{3} l}=C_{1} \\
& \alpha_{3 a}=-\frac{\beta_{3}}{2}=C_{2} \\
\gamma= & \frac{u_{c}\left(1-\beta_{3}\right)}{\left(u_{c}+(p-i q)\right)} e^{-\lambda_{3} l} \\
+ & \frac{\beta_{3}\left(u_{c}-(p-i q)\right)}{2\left(u_{c}+(p-i q)\right)} e^{-2 \lambda_{3} l} \\
+ & \frac{\beta_{3}}{2}=C_{1}-C_{2}=C_{3} .
\end{aligned}
$$

Note that the variables $a, p, q, \lambda, \mu, C_{1}, C_{2}$ and $C_{3}$ are defined the same as those in Xia et al (2007). Equations (B1c) and (B1d) are identical to the corresponding terms in the head solutions of Xia et al (2007, equations A9 and A10). Note that the complex expression used in their paper is $\operatorname{Re}\left(e^{i \omega t}\right)$ while that used in this study is $\operatorname{Re}\left(e^{-i \omega t}\right)$.

\section{B2. Ignoring outlet-capping effect in offshore aquifer}

When $m \rightarrow 0$ and $T_{o} \rightarrow 1$, the variables $\alpha_{1}, \alpha_{2}, \alpha_{3}$, and $\alpha_{4}$ become $\alpha_{1 b}, \alpha_{2 b}, \alpha_{3 b}$, and $\alpha_{4 b}$, respectively. Based on equations (A6a-s), one can obtain where variables $\Gamma_{1}$ and $\Gamma_{2}$ are respectively defined as:

$$
\begin{aligned}
\Gamma_{1}= & e^{-\lambda_{3} l}\left(\beta_{1} \lambda_{1}-\beta_{2} \lambda_{2}-\beta_{1} \lambda_{3}+\beta_{2} \lambda_{3}\right) \\
& -\Gamma_{2} e^{\lambda_{3} l}
\end{aligned}
$$

and

$$
\Gamma_{2}=\beta_{1} \lambda_{1}-\beta_{2} \lambda_{2}+\beta_{1} \lambda_{3}-\beta_{2} \lambda_{3} .
$$

Therefore, equations (3a) and (3b) can be respectively written as:

$$
\begin{aligned}
h_{1}(x, t)= & h_{\mathrm{MSL}}+\operatorname{Re}\left[A _ { 0 } \left(\alpha_{1 b} e^{-\lambda_{1} x}\right.\right. \\
& \left.\left.+\alpha_{2 b} e^{-\lambda_{2} x}\right) e^{-i \omega t}\right] \\
h_{2}(x, t)= & h_{\mathrm{MSL}}+\operatorname{Re}\left[A _ { 0 } \left(\alpha_{1 b} \beta_{1} e^{-\lambda_{1} x}\right.\right. \\
& \left.\left.+\alpha_{2 b} \beta_{2} e^{-\lambda_{2} x}\right) e^{-i \omega t}\right]
\end{aligned}
$$

and equation (3c) becomes

$$
\begin{aligned}
h_{2}(x, t)= & h_{\mathrm{MSL}}+\operatorname{Re}\left[A _ { 0 } \left(\alpha_{3 b} e^{\lambda_{3} x}\right.\right. \\
& \left.\left.+\alpha_{4 b} e^{-\lambda_{3} x}+\beta_{3}\right) e^{-i \omega t}\right] .
\end{aligned}
$$

Equations (B2g-i) are exactly the same as the solutions presented in Chuang and Yeh (2008, equations $10 \mathrm{a}-\mathrm{c}$ ).

$$
\begin{gathered}
\alpha_{1 b}=\frac{e^{-\lambda_{3} l}\left(-\beta_{2} \lambda_{2}+\beta_{2} \lambda_{3}-\beta_{3} \lambda_{3}\right)+e^{\lambda_{3} l}\left(\beta_{2} \lambda_{2}+\beta_{2} \lambda_{3}-\beta_{3} \lambda_{3}\right)-2 \lambda_{3}\left(1-\beta_{3}\right)}{\Gamma_{1}} \\
\alpha_{2 b}=\frac{e^{-\lambda_{3} l}\left(\beta_{1} \lambda_{1}-\beta_{1} \lambda_{3}+\beta_{3} \lambda_{3}\right)+e^{\lambda_{3} l}\left(-\beta_{1} \lambda_{1}-\beta_{1} \lambda_{3}+\beta_{3} \lambda_{3}\right)+2 \lambda_{3}\left(1-\beta_{3}\right)}{\Gamma_{1}} \\
\alpha_{3 b}=\frac{e^{\lambda_{3} l}\left(-\beta_{1} \beta_{2} \lambda_{1}+\beta_{1} \beta_{3} \lambda_{1}+\beta_{1} \beta_{2} \lambda_{2}-\beta_{2} \beta_{3} \lambda_{2}\right)-\left(1-\beta_{3}\right)\left(-\beta_{1} \lambda_{1}+\beta_{2} \lambda_{2}+\beta_{1} \lambda_{3}-\beta_{2} \lambda_{3}\right)}{\Gamma_{1}} \\
\alpha_{4 b}=\frac{e^{-\lambda_{3} l}\left(\beta_{1} \beta_{2} \lambda_{1}-\beta_{1} \beta_{3} \lambda_{1}-\beta_{1} \beta_{2} \lambda_{2}+\beta_{2} \beta_{3} \lambda_{2}\right)-\left(1-\beta_{3}\right) \Gamma_{2}}{\Gamma_{1}}
\end{gathered}
$$




\section{B3. Extending roof length of offshore aquifer to infinity}

If $T_{o} \rightarrow 1$ and the roof length of the offshore aquifer extends to infinity (i.e., $l \rightarrow \infty$ ) the variables $\alpha_{1}$, $\alpha_{2}, \alpha_{3}$, and $\alpha_{4}$ of equations (A6a-d) reduce to $\alpha_{1 c}$, $\alpha_{2 c}, \alpha_{3 c}$, and $\alpha_{4 c}$, respectively, and

$$
\begin{gathered}
\alpha_{1 c}=\frac{-\beta_{2} \lambda_{2}-\beta_{2} \lambda_{3}+\beta_{3} \lambda_{3}}{\Gamma_{2}} \\
\alpha_{2 c}=\frac{\beta_{1} \lambda_{1}+\beta_{1} \lambda_{3}-\beta_{3} \lambda_{3}}{\Gamma_{2}} \\
\alpha_{3 c}=\frac{\beta_{1} \beta_{2} \lambda_{1}-\beta_{1} \beta_{3} \lambda_{1}-\beta_{1} \beta_{2} \lambda_{2}+\beta_{2} \beta_{3} \lambda_{2}}{\Gamma_{2}}
\end{gathered}
$$

and

$$
\alpha_{4 c}=0 .
$$

Equations (B3a-c), expressed as the dimensionless parameters, are in fact the same as the corresponding terms defined in Chuang and Yeh (2007, equations 13-15).

\section{B4. Ignoring both outlet-capping and roof length}

If $l \rightarrow 0$ and $m \rightarrow 0$, the effect of outlet-capping is negligible and the roof does not extend under the sea. These two conditions are also used in Jeng et al (2002). Accordingly, the variables $\alpha_{1}$ and $\alpha_{2}$ become $\alpha_{1 d}$ and $\alpha_{2 d}$, respectively. Based on equations (B2a) and (B2b), one can obtain

$$
\begin{gathered}
\alpha_{1 b}=\frac{\beta_{2}-1}{\beta_{2}-\beta_{1}}=-\frac{T_{1} \lambda_{2}^{2}+i S_{1} \omega}{T_{1}\left(\lambda_{1}^{2}-\lambda_{2}^{2}\right)}=\alpha_{1 d} \\
\alpha_{2 b}=\frac{1-\beta_{1}}{\beta_{2}-\beta_{1}}=\frac{T_{1} \lambda_{1}^{2}+i S_{1} \omega}{T_{1}\left(\lambda_{1}^{2}-\lambda_{2}^{2}\right)}=\alpha_{2 d} \\
\beta_{1}=1-\frac{S B_{1}}{2 a_{1}^{2} u_{i}}-\frac{S i}{u_{i}}=1-\frac{T_{1} \lambda_{1}^{2}}{L_{i}}-\frac{i S_{1} \omega}{L_{i}} \\
\beta_{2}=1-\frac{S B_{2}}{2 a_{1}^{2} u_{i}}-\frac{S i}{u_{i}}=1-\frac{T_{1} \lambda_{2}^{2}}{L_{i}}-\frac{i S_{1} \omega}{L_{i}} .
\end{gathered}
$$

Equations (B4a) and (B4b) can be found in Jeng et al (2002, equation 14). In addition, equations (3a) and (3b) are exactly the same as the ones of Jeng et al (2002, equations 12 and 13) except that the variables of $\beta_{1}$ and $\beta_{2}$ are in terms of dimensionless parameters.

\section{References}

Chuang M H and Yeh H D 2007 An analytical solution for the head distribution in a tidal leaky aquifer extending an infinite distance under the sea; Adv. Water Resour. 30(3) 439-445.

Chuang M H and Yeh H D 2008 Analytical solution for tidal propagation in a leaky aquifer extending finite distance under the sea; J. Hydraul. Eng., ASCE 134(4) 447-454.

Chuang M H, Huang C S, Li G H and Yeh H D 2010 Groundwater fluctuations in coastal heterogeneous aquifer systems; Hydrol. Earth Syst. Sci. 14 1819-1826.

Domenico P A and Schwartz F W 1997 Physical and Chemical Hydrogeology; 2nd edn, John Wiley and Sons, New York.

Ferris J G 1951 Cyclic fluctuations of water level as a basis for determining aquifer transmissibility; IAHS Publ. 33 $148-155$.

Geng X, Li H L, Boufadel M C and Liu S 2009 Tide-induced head fluctuations in a coastal aquifer: Effects of the elastic storage and leakage of the submarine outlet-capping; Hydrogeol. J. 17(5) 1289-1296.

Guo Q N, Li H L, Boufadel M C, Xia Y Q and Li G H 2007 Tide-induced groundwater head fluctuation in coastal multi-layered aquifer systems with a submarine outlet-capping; Adv. Water Resour. 30 1746-1755.

Jacob C E 1950 Flow of groundwater; In: Engineering Hydraulics (ed.) Rouse H, John Wiley, New York.

Jeng D S, Li L and Barry D A 2002 Analytical solution for tidal propagation in a coupled semi-confined/phreatic coastal aquifer; Adv. Water Resour. 25(5) 577-584.

Jiao J J and Tang Z 1999 An analytical solution of groundwater response to tidal fluctuation in a leaky confined aquifer; Water Resour. Res. 35(3) 747-751.

Li H and Jiao J J 2001a Analytical studies of groundwaterhead fluctuation in a coastal confined aquifer overlain by a semipermeable layer with storage; Adv. Water Resour. 24(5) 565-573.

Li H and Jiao J J 2001b Tide-induced groundwater fluctuation in a coastal leaky confined aquifer system extending under the sea; Water Resour. Res. 37(5) 1165-1171.

Li H L, Li G, Cheng J and Boufadel M C 2007 Tide-induced head fluctuations in a confined aquifer with sediment covering its outlet at the sea floor; Water Resour. Res. 43(3) W03404.

Li G H, Li H L and Boufadel M C 2008 The enhancing effect of the elastic storage of the seabed aquitard on the tide-induced groundwater head fluctuation in confined submarine aquifer systems; J. Hydrol. 350(1-2) 83-92.

Van der Kamp G 1972 Tidal fluctuations in a confined aquifer extending under the sea; 24th International Geological Conference, Montreal (ed.) J E Gill, Section 11 101-106.

Xia Y, Li H L, Boufadel M C, Guo Q and Li G 2007 Tidal wave propagation in a coastal aquifer: Effects of leakages through its submarine outlet-capping and offshore roof; J. Hydrol. 337 249-257. 